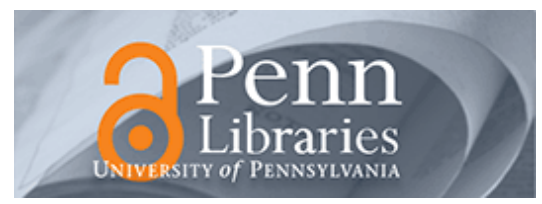

University of Pennsylvania ScholarlyCommons

June 2002

\title{
Individual QoS versus Aggregate QoS: A Loss Performance Study
}

\author{
Ying $\mathrm{Xu}$ \\ University of Pennsylvania \\ Roch A. Guérin \\ University of Pennsylvania, guerin@acm.org
}

Follow this and additional works at: https://repository.upenn.edu/ese_papers

\section{Recommended Citation}

Ying Xu and Roch A. Guérin, "Individual QoS versus Aggregate QoS: A Loss Performance Study", . June 2002.

Copyright 2002 IEEE. Reprinted from Proceedings of the Twenty-First Annual Joint Conference of the IEEE Computer and Communication Societies 2002 (INFOCOM 2002), Volume 3, pages 1170-1179.

Publisher URL: http://ieeexplore.ieee.org/xpl/tocresult.jsp?isnumber=21923\&isYear=2002

This material is posted here with permission of the IEEE. Such permission of the IEEE does not in any way imply IEEE endorsement of any of the University of Pennsylvania's products or services. Internal or personal use of this material is permitted. However, permission to reprint/republish this material for advertising or promotional purposes or for creating new collective works for resale or redistribution must be obtained from the IEEE by writing to pubs-permissions@ieee.org. By choosing to view this document, you agree to all provisions of the copyright laws protecting it.

This paper is posted at ScholarlyCommons. https://repository.upenn.edu/ese_papers/83

For more information, please contact repository@pobox.upenn.edu. 


\title{
Individual QoS versus Aggregate QoS: A Loss Performance Study
}

\begin{abstract}
This papers explores, primarily by means of analysis, the differences that can exist between individual and aggregate loss guarantees in an environment where guarantees are only provided at an aggregate level. The focus is on understanding which traffic parameters are responsible for inducing possible deviations and to what extent. In addition, we seek to evaluate the level of additional resources, e.g., bandwidth or buffer, required to ensure that all individual loss measures remain below their desired target. The paper's contributions are in developing analytical models that enable the evaluation of individual loss probabilities in settings where only aggregate losses are controlled, and in identifying traffic parameters that play a dominant role in causing differences between individual and aggregate losses. The latter allows the construction of guidelines identifying what kind of traffic can be safely multiplexed into a common service class.
\end{abstract}

\section{Keywords}

Network, QoS, Aggregate

\section{Comments}

Copyright 2002 IEEE. Reprinted from Proceedings of the Twenty-First Annual Joint Conference of the IEEE Computer and Communication Societies 2002 (INFOCOM 2002), Volume 3, pages 1170-1179.

Publisher URL: http://ieeexplore.iee. $.0 \mathrm{rg} / \mathrm{xpl} /$ tocresult.jsp?isnumber=21923\&isYear=2002

This material is posted here with permission of the IEEE. Such permission of the IEEE does not in any way imply IEEE endorsement of any of the University of Pennsylvania's products or services. Internal or personal use of this material is permitted. However, permission to reprint/republish this material for advertising or promotional purposes or for creating new collective works for resale or redistribution must be obtained from the IEEE by writing to pubs-permissions@ieee.org. By choosing to view this document, you agree to all provisions of the copyright laws protecting it. 


\title{
Individual QoS versus Aggregate QoS: A Loss Performance Study
}

\author{
Ying $\mathrm{Xu}$ and Roch Guérin \\ Department of Electrical Engineering, University of Pennsylvania, Philadelphia, PA, 19104 \\ E-mail: (yingx,guerin)@ee.upenn.edu
}

\begin{abstract}
This papers explores, primarily by means of analysis, the differences that can exist between individual and aggregate loss guarantees in an environment where guarantees are only provided at an aggregate level. The foc us is on understanding which traffic parameters are responsible for inducing possible deviations and to what extent. In addition, we seek to evaluate the level of additional resources, e.g., bandwidth or buffer, required to ensure that all individual loss measures remain below their desired target. The paper's contributions are in developing analytical models that enable the evaluation of individual loss probabilities in settings where only aggregate losses are controlled, and in identifying traffic parameters that play a dominant role in causing differences between individual and aggregate losses. The latter allows the construction of guidelines identifying what kind of traffic can be safely multiplexed into a common service class.
\end{abstract}

\section{INTRODUCTION}

The provision of QoS guarantees is by now an extensively investigated and reasonably well understood topic. The literature abounds with algorithms for enforcing different levels of service and results evaluating their respective performance, see, e.g., [9] for a recent survey. Similarly, technology is now available that implements sophisticated QoS capabilities, see, e.g., [13]. However, despite all this progress, the deployment of QoS capabilities in operational networks has been by most accounts slow. Many factors have conspired to this, but one of them has been a recurring theme in discussions aimed at understanding the reasons behind this slow pace. Specifically, the complexity of managing a broad range of fine grain (individual) QoS requirements across a network of the scale of the Internet is a daunting task. As a result, there has been a renewed interest in designing scalable QoS solutions.

There have been two main directions aimed at developing scalable QoS solutions. The first, embodied in works such as [20], [21], [24], targets the emulation of fine-grain QoS solutions without requiring per flow information. The second, represented by proposals such as Diff-Serv [3], relies on coarsening the different levels of QoS that the network offers into a small number of service classes. Our focus is on this latter class of solutions.

Limiting the number of service classes that the network offers clearly improves scalability. However, this comes at a cost, namely the lack of awareness of the exact level of performance that an individual user/application experiences. In other words, implicit in the use of service classes is the assumption that the

This work was supported in part through NSF grant ANI00-85930. users aggregated into a given service class all experience the same level of service, or a least a level of service better than the desired target for the service class. The extent to which this is true certainly depends on the level of sophistication used when making decisions on whether or not to accept additional traffic. In particular, there has been extensive work on call admission procedures aimed at deciding if and when to accept a new request. The methods they use vary and rely either on models for computing the amount of resources required by a given request, e.g., [8], [5], or on some combinations of measurements and traffic characterization, e.g., [6], [7], [12]. However, they typically require some flow level awareness, e.g., in the form of traffic parameters that are signalled to the network when the request is made. Our focus is somewhat different in that we assume that provisioning is the primary tool used to monitor network performance and make decisions on whether additional traffic can be accommodated.

By its nature and in order to ensure its scalability, monitoring is typically done at an aggregate level, e.g., the losses experienced by a service class. In that context, our objective is to gain a better understanding of how such aggregate measures map into individual performance, and in particular determine if specific guidelines can be formulated to avoid situations where the two differ significantly. For example, such guidelines could specify which range of traffic can be safely multiplexed in the same service class. Note that such recommendations would obviously be formulated in terms of traffic parameters. However, contrary to an environment where call admission is used to dynamically make decisions on accepting new traffic, these would be primarily used for making off-line decisions on how to assign different traffic types to service classes.

In this initial study, we focus on a specific performance measure, namely, the packet loss probability. Our approach is primarily analytical, although we also rely on simulations to investigate certain configurations where analysis is difficult, if not impossible. Our main thrust is to develop a number of new models or extensions to existing models, which allow us to evaluate the loss probability experienced by an individual flow when only the overall loss probability of the service class to which this flow belongs is observable. This enables us to evaluate the influence of the different traffic parameters of a flow, e.g., its peak rate, average rate, burst duration, etc., on the potential deviation between its own loss probability and the overall loss probability. In that context, the identification of parameters that can result in significant deviations is of special 
interest, as it can be translated into guidelines for safely aggregating flows. In addition, we also investigate the sensitivity of any performance deviation in terms of the amount of additional resources, i.e., bandwidth or buffer, required to ensure that even the worst performer in a service class experiences a level of performance equal to or better than the desired target for the service class.

We believe that the results of this paper provide an important tool to assess the feasibility and limitations of aggregate QoS solutions, and improve our understanding of how to use them. The rest of this paper is structured as follows. Section II introduces and motivates the different models and systems assumptions. Analytical expressions of individual loss probabilities are provided for those models in Section III, with most of the derivations being relegated to an extended version of the paper available on-line [23]. Sections IV and V report results obtained from the models of Section III. Finally, Section VII summarizes the main findings of the paper and their implications for aggregate QoS solutions.

\section{Model And Methodology}

In this section, we describe the model and methodology we rely on to investigate the behavior of individual loss probabilities in an environment based on aggregate service classes. This includes the traffic model we assume for users, the different service configurations we analyze, and how we measure differences between individual and aggregate loss probabilities. Specifically, the system we consider is that of a single server, finite buffer, FIFO queue that represents the queue associated with a given service class on a network link. Parameters that influence our ability to analyze such a system include the statistics of the traffic generated by individual users, as well as the total number of users that are aggregated into this single queue.

\section{A. Input Traffic Model}

We consider two different models for characterizing the traffic generated by an individual user. The first is a standard ONOFF Markov source [1], with exponentially distributed ON and OFF periods and a fixed transmission rate when ON (active). Such a source can be described using a 3-tuple $\langle R, b, \rho\rangle$, where $R$ is the transmission (peak) rate when the source is active, $b$ is the average duration of an active or ON period, and $\rho$ represents the fraction of time the source is active, or its utilization. The rationale for such a source model is both that it lends itself to the development of tractable analytical models from which intuition and insight can be derived, and that its simple three parameters description can be easily mapped onto popular traffic control devices such as leaky buckets, e.g., see [11] for a discussion on this issue. As a result, it captures the behavior of configurations in which performance is mainly determined by "burst-level" congestion. This will be the case when provisioning used for the service class allows for periods of time during which the incoming traffic exceeds the allocated capacity.

The second model considered is the $\sum D_{i} / D / 1$ queue [22], [18], [17]. In this model, each source is assumed to be constant rate and to periodically (every $D_{i}$ units of time) generate a single, unit size ${ }^{1}$ packet. Sources can differ in terms of both their

\footnotetext{
${ }^{1}$ Its transmission takes one unit of time
}

period and the position at which they generate their packet in a period. The choice of the position at which a given source generates its packet within a period is assumed to be independent of that of other sources, and to be drawn from a uniform distribution over the interval $\left[0, D_{i}\right)$. This second model captures a different environment and system configuration from the first one in which congestion primarily occurs at the packet level. This is the case when provisioning is done based on the "worstcase" assumptions regarding the traffic that a user can generate. For example, this could apply to provisioning rules used to support a constant rate, low delay service class based on the Diff-Serv Expedited Forwarding Per Hop Behavior (PHB) [4], or representative of a network that relies on conservative (peak rate) provisioning.

We believe that these two traffic models, because of their differences, bracket a broad and realistic range of possible configurations. When combined with other various combinations of system parameters as described below, they should provide a reasonably comprehensive investigation of the problem space.

\section{B. System Parameters and Performance Measures}

In developing models to explore possible deviations between individual and aggregate loss probabilities, we consider several configurations that sample the range of possible environments.

The first parameter we vary is the number of users aggregated in the same service class, i.e., the number of distinct traffic sources multiplexed in the FIFO queue. In particular, we focus on two cases: a two-source configuration and a "manysource" one. We select these two configurations not only because they are amenable to analysis, but also because they correspond to different boundary conditions, i.e., an environment where few large bandwidth connections share resources, and one where many small (compared to the link capacity) flows are multiplexed into the same queue. We expect these two environments to exhibit different sensitivity to the traffic parameters of individual sources, and to possibly yield deviations between individual and aggregate losses of different magnitude.

Another system parameter we consider is the size of the FIFO queue into which flows are multiplexed. In particular, we consider both the cases of bufferless (buffer size of zero) and buffered systems. In many instances, bufferless systems lend themselves to more tractable analysis, while qualitatively capturing performance trends. In cases where models are limited to bufferless systems, we also rely on simulations to extend the investigation to buffered systems. In all cases of interest, the simulation results confirmed the trends observed from the bufferless analytical results.

Our focus is to derive explicit expressions, function of source and system parameters, for both individual and aggregate loss probabilities in different system configurations that we consider. Loss probabilities correspond to the ratio of the total number of bits lost to the total number of bits sent by either an individual user or all users. We denote the overall loss probability $P_{L}$, and the loss probability of user $i$ as $P_{L}^{i}$. $P_{\max }^{N}$ corresponds to the maximum loss probability experienced by an individual user (user $N$ ) in the service class. Note that traditional models, e.g., [1], [19], have focused on expressions for 
the (overall) overflow probability rather than the loss probability. The latter is in our mind a more realistic measure of the performance that users experience. As outlined in Section III, deriving expressions for the loss rather than the overflow probability in a finite buffer system calls for some slight modifications to the derivations.

The expressions of individual and aggregate loss probabilities allow us to investigate cases when they potentially differ. We carry out these investigations by first selecting a target loss probability $P_{\max }$ for the service class, and then computing the \{minimum amount of bandwidth $C$ needed to ensure an overall loss probability $P_{L} \leq P_{\max }$. We then identify the user that experiences the highest individual loss probability $P_{\max }^{N}$, and compute the ratio $P_{\max }^{N} / P_{L}$. Note that for simplicity, in most cases we limit our investigations to configurations that only involve two types of users. This facilitates the identification of which user (type) experiences the higher losses. In addition to computing the ratios between individual and aggregate losses, we also evaluate the percentage by which the bandwidth allocated to the service class needs to be increased to ensure $P_{\max }^{N} \leq P_{\max }$. In other words, if $C_{N}$ denotes the minimum amount of bandwidth needed so that $P_{\max }^{N} \leq P_{\max }$, we evaluate the quantity $\left(C_{N}-C\right) / C$. This serves as a useful metric to evaluate the penalty that an aggregate service model incurs.

\section{Analytical Results}

This section is devoted to the presentation of the analytical results that allow us to compute and compare both individual and aggregate loss probabilities. Proofs and additional details are available from an extended version technical report [23]. Four distinct models are presented in this section. The first two correspond to the two-source cases of both ON-OFF and periodic sources, and the last two correspond to the "many-source" scenario, again for ON-OFF and periodic sources.

\section{A. The Two On-Off Sources Case}

Since there is conceptually little difference in the derivation of expressions for a two-source system or an $N$-source system, we proceed to derive general expressions for an $N$-source system, from which we subsequently specialize to the two-source system by letting $N$ equal to 2 . The analysis of systems that involve Markov modulated traffic sources is by now a mature area, e.g., [1], [15], [19], and we rely on this existing body of work to develop our model. The main difference between those works and ours is that ours focus on loss as opposed to overflow probability and, most important, on evaluating both individual and aggregate performance.

Our initial model consists of $N$ independent ON-OFF fluid sources that feed an infinite buffer, single server queue. Source $i$ is characterized by the 3-tuple described in Section II-A, denoted by $\left(R_{i}, b_{i}, \rho_{i}\right)$. The input process to the buffer can then be described through a state vector: $\mathbf{S}=\left(s_{1}, s_{2}, \cdots, s_{N}\right)$, where $s_{i}$ is 0 when source $i$ is OFF and 1 when it is ON. For any state, the input rate $\gamma_{S}$ to the system is given by $\gamma_{\mathbf{S}} \doteq \mathbf{S} \cdot \mathbf{R}^{\mathbf{T}}$, where $\mathbf{R}=\left(R_{1}, R_{2}, \cdots, R_{N}\right)$ is the peak rate vector of the sources. If the stationary probability that the input is in state $\mathbf{S}$ is denoted by $\pi_{\mathbf{S}}$, under the standard assumption that the system is ergodic the stationary loss probability experienced by source $i$ in a finite buffer system of size $x$ can then be approximated by:

$$
P_{L}^{i}=\frac{\sum_{\substack{\mathbf{S}:\left(s_{i}=1, \gamma_{\mathbf{S}}>C\right)}}\left(\pi_{\mathbf{S}}-F_{\mathbf{S}}(x)\right)\left(R_{i}-C \cdot \frac{R_{i}}{\gamma_{\mathbf{S}}}\right)}{\rho_{i} R_{i}}=\frac{r_{L}^{i}}{r_{S}^{i}},
$$

where $r_{L}^{i}$ and $r_{S}^{i}$ correspond to the long term loss rate and sending rate of source $i$. The quantity $F_{\mathbf{S}}(x)$ is the stationary probability that the queue length is smaller than $x$ and the system is in state $\mathbf{S}$, which can be readily obtained from results of either [15] or [19].

Similarly, the overall loss rate $P_{L}$ can be expressed as:

$$
P_{L}=\frac{\sum_{\mathbf{S}: \gamma_{\mathbf{S}}>C}\left(\pi_{\mathbf{S}}-F_{\mathbf{S}}(x)\right)\left(\gamma_{\mathbf{S}}-C\right)}{\sum_{i}^{N} \rho_{i} R_{i}}=\frac{r_{L}}{r_{S}},
$$

where $r_{L}$ and $r_{S}$ correspond to the overall long term loss rate and sending rate, respectively.

Expressions for individual and aggregate loss probabilities can be readily obtained from equations (1) and (2) for the twosource case simply by letting $N=2$. For example, in the case where $R_{1} \leq C, R_{2} \leq C$, and $R_{1}+R_{2}>C$, i.e., losses occur only when both sources are active, we will have:

$$
\begin{gathered}
P_{L}^{1}=\frac{\left(\pi_{(1,1)}-F_{(1,1)}(x)\right) \cdot\left(R_{1}+R_{2}-C\right)}{\rho_{1}\left(R_{1}+R_{2}\right)} \\
P_{L}^{2}=\frac{\left(\pi_{(1,1)}-F_{(1,1)}(x)\right) \cdot\left(R_{1}+R_{2}-C\right)}{\rho_{2}\left(R_{1}+R_{2}\right)},
\end{gathered}
$$

where $\pi_{(1,1)}$ is the stationary probability that both sources are active. As we shall discuss further in Section IV, the simple form of equation (3) helps explicitly identify the impact of different parameters. In particular, we see that for this special case, the ratio of individual loss probabilities is equal to the ratio of the utilizations of the respective sources. In other words, the source with the lower utilization will see a proportionally higher loss probability. This simple but nevertheless interesting observation is one that will be subsequently confirmed in other and more general configurations.

\section{B. The Two Periodic Sources Case}

In this section, we consider the special case of the $\sum D_{i} / D / 1$ queue with only two sources of periods $D_{1}$ and $D_{2}$, respectively, i.e., the $\left(D_{1}+D_{2}\right) / D / 1$ queue. For this model, we assume a bufferless system, since having a buffer of size larger than or equal to even one packet will eliminate all losses. In spite of its extreme simplicity, this system is again useful because of the insight it provides. For this simple system, the following proposition can be shown to hold.

Proposition 1: For a bufferless $\left(D_{1}+D_{2}\right) / D / 1$ queue, where $D_{1}, D_{2}$ are integers and $2 \leq D_{1} \leq D_{2}$, the loss probability ratios $P_{L}^{2} / P_{L}^{1}$ and $P_{L}^{2} / P_{L}$ satisfy:

$$
\frac{P_{L}^{2}}{P_{L}^{1}}=\frac{D_{2}}{D_{1}} \quad \frac{P_{L}^{2}}{P_{L}}=\frac{1+\frac{D_{2}}{D_{1}}}{2}
$$


Moreover, if $D_{1}$ can be divided by $D_{2}$, then:

$$
P_{L}^{1}=\frac{1}{D_{2}} \quad P_{L}^{2}=\frac{1}{D_{1}} \quad P_{L}=\frac{2}{D_{1}+D_{2}}
$$

Proof: The proof is given in [23].

The above proposition, though simple, states that the source with the larger period sees greater losses in a proportion that is in the ratio of its period to that of the other source. This is an observation similar to the one made based on equation (3) for two ON-OFF sources, which will occur in several other configurations, as we will see later.

\section{The Many ON-OFF Sources Case}

Equations (1) and (2) were derived for the general case of $N$ sources, but as $N$ grows large, the required computations quickly become prohibitive because of the well-known "state explosion", and this makes numerical evaluation difficult if not impossible. As a result, we rely on simulations for evaluating buffered systems, and shift the focus of our analysis to a bufferless system for which numerical evaluation remains feasible. A bufferless model is a reasonable approximation when a large number of sources are multiplexed. In this case, the input traffic can be well approximated using the general technique of rate envelope multiplexing [17, Section 4.1.1], and data loss can then be characterized by relying on the fact that it only occurs when the input rate (envelope) exceeds the available service rate. Under these assumptions, models already exist [16] that give explicit expressions for the quantities of interest in the context of this paper, i.e., individual and aggregate loss probabilities. For completeness, we briefly restate the relevant results and assumptions of [16], and identify the minor generalization we introduce. Note that [16] is one of the first works to explicitly target understanding when and why differences in performance can arise when aggregating many different types of sources. Some of its results are consistent with those we derive in this paper, and the main differences are both in terms of the broader investigation we undertake, and more important, of our focus on explicitly identifying the impact of individual traffic parameters across many different scenarios.

In the bufferless system we consider, the total input traffic is divided into two parts: the background traffic and the traffic associated with a specific source. This source (without loss of generality, we assume it is source $N$ ) is the one we focus on, and whose traffic parameters we vary. We denote $\lambda_{t}, \lambda_{t}^{b}$ and $\lambda_{t}^{N}$ as the random variables associated with the rate envelop at time $t$ of the total traffic, the background traffic, and the rate of source $N$ respectively. Similarly, the variables $m, m_{b}$, and $m_{N}$ identify the corresponding mean rates. Under those assumptions, the overall loss probability $P_{L}$ and the loss probability $P_{L}^{N}$ of source $N$ can be obtained from a minor generalization of the results of [16], by assuming a link capacity of $C$ instead of a unit link capacity, and by expanding the expression for $P_{L}$ simply by conditioning on $\lambda_{t}^{N}$.

$$
P_{L}^{N}=\frac{E\left[\left(\lambda_{t}-C\right)^{+} \cdot \frac{\lambda_{t}^{N}}{\lambda_{t}}\right]}{\rho_{N} * R_{N}}
$$

$$
=E\left[\left(\frac{\lambda_{t}^{b}+R_{N}-C}{\lambda_{t}^{b}+R_{N}}\right)^{+}\right],
$$

and

$$
\begin{aligned}
P_{L} & =\frac{E\left[\left(\lambda_{t}-C\right)^{+}\right]}{m} \\
& =\frac{\rho_{N} E\left[\left(\lambda_{t}^{b}+R_{N}-C\right)^{+}\right]+\left(1-\rho_{N}\right) E\left[\left(\lambda_{t}^{b}-C\right)^{+}\right]}{m}
\end{aligned}
$$

The expectations in both equations (6) and (7) can be evaluated numerically if $\lambda_{t}^{b}$ is explicitly specified. In most of our tests, the background traffic consists of homogeneous sources, as we focus on the impact of varying the traffic parameters of source $N$, so that $\frac{\lambda_{t}^{b}}{R_{b}}$ is simply a binomial distribution.

\section{The Many Periodic Sources Case}

The overflow probability for the $\sum D_{i} / D / 1$ queue has been investigated in [22], [18], [17]. Its derivation is based on the Bene $\breve{~ a p p r o a c h ~ t h a t ~ i s ~ e x t e n s i v e l y ~ d o c u m e n t e d ~ i n ~[17] . ~ I n ~ t h i s ~}$ paper, we build on and extend the methods of [22] and [18] to obtain upper and lower bounds for individual loss probabilities. We briefly outline the model assumptions and state the final results, while details and proofs can be found in [23].

The model we consider consists of $J$ different types of sources. There are $N_{j}$ independent sources of type $j, 1 \leq$ $j \leq J$, each with a period of $D_{j}$ time units. The individual source we focus on is source $i$ with period $D_{i}$. In order to ensure that the system is stationary and ergodic, we further impose the condition that the total load $\rho$ is less than 1, i.e., $\rho=1 / D_{i}+\sum_{j=1}^{J} \frac{N_{j}}{D_{j}}<1$. The queue length or backlog in the system at time $t$ is denoted as $V_{t}$, and we use the expression

$$
\operatorname{Pr}\left\{V_{0}>x \mid \text { One arrival from source } D_{i} \text { at } 0^{+}\right\}
$$

to approximate the loss probability of source $D_{i}$ in a finite buffer system of size $x$. In [23], the following lower and upper bounds are established:

$$
\begin{gathered}
P_{L}^{i} \geq \sum_{\substack{n>x \\
n \neq x+\left\lceil N D_{i}\right\rceil-1}}(1-\rho) \cdot\left(\sum_{\substack{K(n, x) \\
=n-d(n, x)}} \prod_{j=1}^{J} q_{j}\left(k_{j}(n, x)\right)\right) \\
P_{L}^{i} \leq \sum_{\substack{n>x \\
n \neq x+\left\lceil N D_{i}\right\rceil-1}}\left\{\sum _ { \substack { K ( n , x ) \\
= n - d ( n , x ) } } \left[\prod _ { j = 1 } ^ { J } q _ { j } \left(k_{j}(n, x) .\right.\right.\right. \\
\left.\left.\left(1-\frac{N_{j}-k_{j}(n, x)}{D_{j}\left(1-p_{j}(n, x)\right)}\right)^{+}\right]\right\},
\end{gathered}
$$

where $d(n, x)=\sum_{j=1}^{J} N_{j} \cdot\left\lfloor\frac{n-x}{D_{j}}\right\rfloor+\left\lfloor\frac{n-x}{D_{i}}\right\rfloor, p_{j}(n, x)=$ $\frac{n-x}{D_{j}}-\left\lfloor\frac{n-x}{D_{j}}\right\rfloor$ and $K(n, x)=\sum_{j=1}^{J} k_{j}(n, x)$. The value of $k_{j}(n, x)$ can vary between 0 and $N_{j}$ and $q_{j}\left(k_{j}(n, x)\right)$ is the probability density function of a binomial distribution with parameter $\left(N_{j}, p_{j}(n, x)\right)$. 
Upper and lower bounds for the overall loss probability $P_{L}$ can be obtained from equations (9) and (8) based on the following relation:

$$
P_{L}=\frac{\sum_{j=1}^{J} \frac{N_{j}}{D_{j}} \cdot P_{L}^{j}+\frac{1}{D_{i}} \cdot P_{L}^{i}}{\sum_{j=1}^{J} \frac{N_{j}}{D_{j}}+\frac{1}{D_{i}}}
$$

The above upper and lower bounds have been numerically evaluated for many different configurations, and found to be consistently very close to each other (see [23] for a couple of illustrative examples). In this paper, we use the upper bound of equation (9) to approximate the individual loss probability $P_{L}^{i}$ of source $i$.

In the next sections, we rely on the various expressions derived in Section III to investigate when and why individual and aggregate loss probabilities differ.

\section{Loss Deviations In The Two-Source Case}

This section is devoted to exploring configurations that involve only two sources. In all our investigations for both this section and subsequent ones, $P_{\max }$ is fixed at a level of $10^{-4}$.

\section{A. Two On-Off Sources}

In this section, we assume that only two ON-OFF sources are multiplexed into a common queue that is served by a constant rate server of speed $C$ bits/sec, where the value of $C$ has been selected to ensure an overall loss probability $P_{L} \leq 10^{-4}$. The traffic parameters of source 1 are kept fixed at $R_{1}=$ $10^{8} \mathrm{bits} / \mathrm{sec}, b_{1}=0.005 \mathrm{sec}$, and $\rho_{1}=0.5$. In all scenarios described in this section, the buffer size of the queue is set equal to the total average burst size of the two sources. Additional experiments were conducted with different buffer sizes, and did not yield drastically different behaviors. The parameters of source 2 are then varied one or more at a time, with its other parameters kept constant and identical to those of source 1. We rely on equations (1) and (2) to evaluate deviations between $P_{L}^{2}$ and $P_{L}$. We omit results related to varying the burst duration as this parameter was found to have no or only minor impact. Note that this doesn't mean the burst duration has no impact. It certainly does, and as it increases, the total allocated bandwidth $C$ increases to accommodate the burstier arrival process of source 2. However, contrary to what happens with the peak rate and the utilization, varying the burst duration while maintaining the aggregate loss probability below the desired target of $P_{L}=10^{-4}$, does not introduce significant differences between the two sources in terms of loss.

A first set of conclusions illustrated by Figure 1 and verified through extensive additional experiments with different configurations, is that individual traffic parameters can indeed induce differences in terms of the loss probability that a given source experiences. In particular, we see that a higher peak rate and a lower utilization (lower rate) both translate into a higher loss probability for source 2 than for source 1 , although the magnitude of the difference is not the same in the two cases.

The fact that both peak rate and utilization can affect the performance of an individual source is reasonably intuitive. A higher peak rate source dumps data faster into the buffer, which

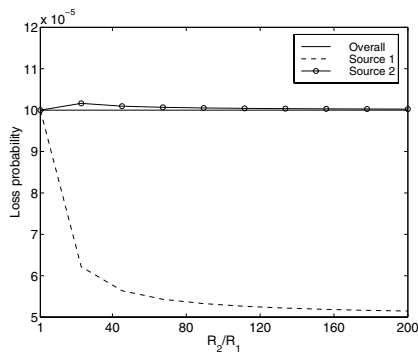

(a) Varying $R_{2}$

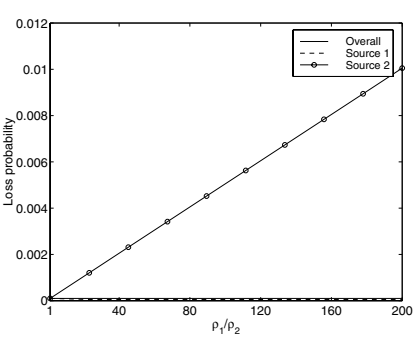

(b) Varying $\rho_{2}$
Fig. 1. Performance Deviations for Two ON-OFF Sources.

increases its likelihood of losing data. Hence, it becomes the dominant contributor to the overall loss, as seen in Figure 1(a), and ends-up experiencing a slightly worse loss probability than the overall. A similar situation arises when the two sources have different utilizations, but because of the potentially much larger magnitude of the difference (see Figure 1(b)), this scenario deserves additional attention.

The main reason why utilization has a greater impact than peak rate is because of how we choose to vary individual parameters and the resulting weight of each source in terms of its traffic contribution. Specifically, as we vary a given parameter of source 2, we keep the others fixed and equal to those of source 1 . When we vary (increase) the peak rate, because the utilization of the two sources remains the same, source 2 endsup being the dominant contributor of traffic into the system. Hence, although it does experience higher losses, because of its higher weight in computing the overall loss probability $P_{L}$, the allocated bandwidth $C$ that is chosen to ensure that $P_{L} \leq 10^{-4}$, also ensures that $P_{L}^{2}$ remains close to this target value.

In contrast, when the utilization of source 2 is varied (decreased) while its peak rate remains identical to that of source 1 , it is source 1 that becomes the dominant traffic contributor. Hence, the allocated bandwidth $C$ is determined based primarily on the performance of source 1 , which allows the loss curve of source 2 to degrade arbitrarily. The reason for this degradation is that the lower utilization of source 2 limits its ability to access the link. This provides source 1 with additional transmission opportunities, which helps to lower its individual loss probability. This is best illustrated through the specific scenario already mentioned in Section III-A, where losses only occur when both sources are active.

In this special case, the individual loss probabilities are given in equation (3), which clearly identifies the impact of the smaller utilization of source 2. Specifically, we have $P_{L}^{2}=$ $P_{L}^{1} \frac{\rho_{1}}{\rho_{2}}$, which increases in a way that is inversely proportional to $\rho_{2}$. This is because losses occur when both sources are active and, therefore, losses are distributed fairly and in proportion to the peak rate of each source, i.e., the individual loss rate $r_{L}^{i}$ is proportional to the peak rate $R_{i}$. Meanwhile, the individual transmission rate $r_{S}^{i}$ is proportional to both the peak rate $R_{i}$ and the utilization $\rho_{i}$. Thus, the effect of the peak rates $R_{i}$ cancel out, and the ratio of the loss probabilities of the two sources is then easily found to be inversely proportional to the ratio of 
their utilizations. A similar trend was also observed in cases where the peak rate of each individual source exceeds the link capacity. However, in such cases a trade-off exists between the impact of the peak rate that influences the losses that a source experiences when it is the only one active, and the utilization that again affects how often both sources are simultaneously active and sharing losses. From our observations, utilization remains the dominant factor, primarily because the allocated bandwidth was typically chosen so as to keep losses at a sufficiently low level when only one source was active.

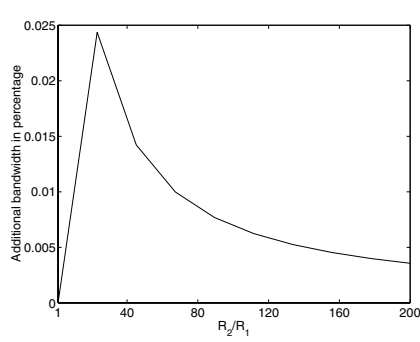

(a) Varying $R_{2}$

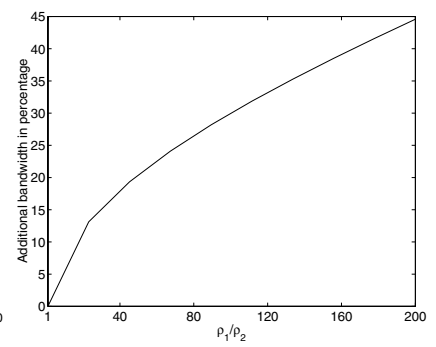

(b) Varying $\rho_{2}$
Fig. 2. Additional Bandwidth for Two ON-OFF Sources.

The next aspect we investigate is the level of additional resources that are required to ensure that both sources experience a loss probability that is below the desired target of $P_{L}=10^{-4}$. The results of this investigation are shown in Figure 2 in the form of the percentage of additional bandwidth required. The figure reports the minimum amount of additional bandwidth needed to ensure adequate performance for both sources. As we can see, Figure 2(b) confirms the potentially severe penalty imposed by mixing sources with very different utilizations. In particular, we see from Figure 2(b) that the amount of additional bandwidth needed can reach about $45 \%$.

In [23], we carried out an additional set of experiments, where we simultaneously varied two instead of only one traffic parameters of source 2 . In particular, we considered scenarios that involved varying $R_{2}$ and $b_{2}, R_{2}$ and $\rho_{2}$, and $b_{2}$ and $\rho_{2}$. The findings from those experiments did not reveal any drastically different behavior from those observed when varying only one parameter at a time. Thus we omit reporting those results here.

\section{B. Two Periodic Sources}

From Proposition 1, we know that as with ON-OFF sources, the source with longer period (smaller rate) experiences higher losses. Similarly, its performance is worse than that of the shorter period source in a way that is proportional to the ratio of their respective periods. The basic reasons for this are essentially the same as those outlined in the case of ON-OFF sources, if the fact that longer period corresponds to smaller utilization is considered.

Moreover, from equation (5), when $D_{1}$ and $D_{2}$ are integers and if $N=D_{2} / D_{1}$ is also an integer, we see that in order to ensure a target loss probability of $\epsilon$, the additional bandwidth in percentage needed (expressed in terms of the corresponding increase of the sources' period) is equal to $\frac{N-1}{2}=\frac{\left(D_{2} / D_{1}-1\right)}{2}$, which is independent of $\epsilon$ and can be made arbitrarily large by increasing $D_{2} / D_{1}$.

\section{Loss Deviations In The Many-Source Case}

This section targets at what one can consider a more realistic set of scenarios, namely, service classes that carry a large number of flows. Such configurations will clearly be more appropriate for high speed links, where one can expect to see just a few service classes, e.g., built on top of a small numbers of Diff-Serv PHBs, each carrying a large number of flows. Our goal, however, remains the same, as we investigate the impact that the individual traffic parameters of a flow can have on the performance it experiences. Intuitively, our expectation is that the presence of a large number of flows is likely to "soften" possible deviations in performance. As we'll see, this intuition will indeed be confirmed.

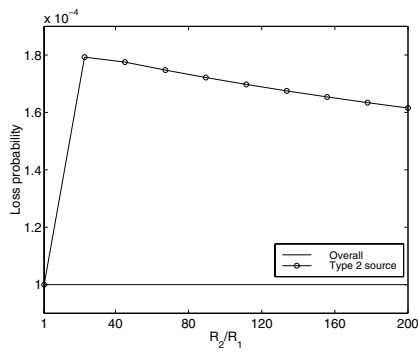

(a) Varying $R_{2}$

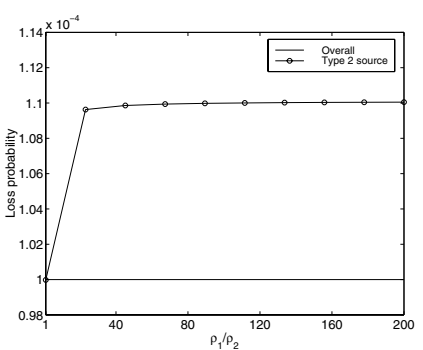

(b) Varying $\rho_{2}$

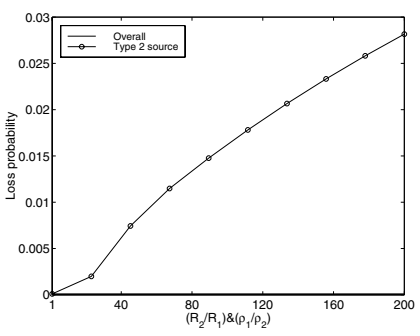

(c) Varying $R_{2}$ and $\rho_{2}$

Fig. 3. Loss Probability Deviations for Many ON-OFF Sources.

\section{A. Many ON-OFF Sources}

In this sub-section, we assume that the input traffic consists of many ON-OFF sources. For simplicity, we limit ourselves to only two types of sources. Sources of type 1 form the "background" traffic, and we assume a total of 1000 such sources, each with a peak rate $R_{1}=10^{5} \mathrm{bits} / \mathrm{sec}$, a burst duration $b_{1}=0.005$ seconds, and a utilization $\rho_{1}=0.5$. As previously mentioned in Section III-C, when the background is traffic is homogenous, the number of active background sources follows a binomial distribution. Using this fact together with the results of a bufferless model in Section III-C, i.e., equations (6) and (7), we evaluate the loss probability experienced by both type 1 sources and (initially) a single type 2 source whose traffic parameters we vary. Note that because the burst duration has no 
impact in a bufferless model, we first limit ourselves to varying the peak rate and the utilization of the type 2 source. This is then followed by scenarios where both $R_{2}$ and $\rho_{2}$ are varied while keeping their product constant, i.e., $R_{2} \rho_{2}=R_{1} \rho_{1}$. Following the methodology of Section IV-A, when any of the parameters of the type 2 source are varied, its other parameters are set to the same values as those of the type 1 sources.

The results are shown in Figure 3. The figure illustrates that variations in either peak rate or utilization alone do yield some differences between the two types of sources. However, those differences are most significant when the type 2 source has both a much higher peak rate and a much lower utilization than the type 1 sources. In particular, Figure 3(c) shows that when $R_{2} / R_{1}=\rho 1 / \rho 2=200$, the loss probability ratio is about 280 .

These results are reasonably intuitive. First, when the peak rate of the type 2 source increases, so does its impact, making it more likely to create congestion when becoming active. The extent to which this also triggers the allocation of additional bandwidth to compensate for this potential increase in losses depends on the impact of the type 2 source on the overall loss. In particular, if its utilization is very low, such contribution will be minor and won't trigger the allocation of any substantial additional bandwidth. Hence, bandwidth allocation is primarily driven by the performance of the type 1 sources, and the higher losses of the type 2 source will remain mostly undetected. This explains why type 2 source can experience losses that are much higher than the aggregate loss when both its peak rate and utilization are varied while keeping its overall data rate constant.

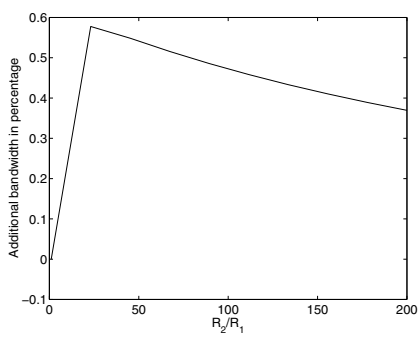

(a) Varying $R_{2}$

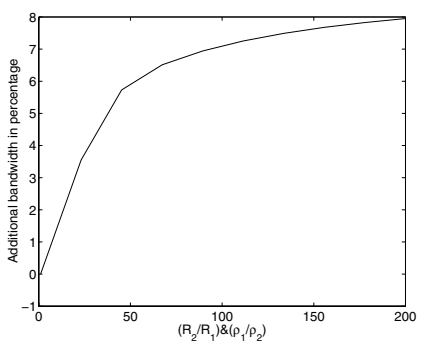

(c) Varying $R_{2}$ and $\rho_{2}$

Fig. 4. Additional Bandwidth for Many ON-OFF Sources.

In contrast, when only the peak rate of the type 2 source is varied (increased), so will its overall data rate. This means that the weight of its losses in the aggregate loss estimate will also increase. This will typically ensure that those losses are properly accounted for in the bandwidth allocation procedure, so that the allocation of additional bandwidth to keep the aggregate loss probability constant is sufficient to also keep the losses of source 2 close to the desired target. This can be observed in Figure 3(a), where the loss probability of the type 2 source is only slightly larger than the overall loss probability. The figure also shows that the relative performance of the type 2 source initially degrades, as its peak rate increases, and then gradually improves. The presence of such "cross-over" point is merely a reflection of the fact that there is a lag between the negative impact of a higher peak rate and its eventual detection by the bandwidth allocation procedure, as its impact on the overall loss probability increases.

Figure 3(b) illustrates that when only the utilization of the type 2 source is varied, the impact on performance differences between the two types of sources is marginal. This is because although its decreasing overall rate means that its weight in the bandwidth allocation procedure becomes less and less, a type 2 source will sample the system in pretty much the same way as a type 1 source due to the fact that its appearance will only contribute a minor increase to the level of congestion. Hence, the two types of sources see mostly the same loss probability.

Because the above results were obtained using a bufferless model, we performed a number of simulations for a buffered system to test our conclusions. A buffer size of 0.5 Mbits, i.e., the total average burst size that can be generated by the background sources, was used. Although we do not report the results due to lack of space, the observed behavior was qualitatively similar as with the bufferless model. In particular, we verified that the burst duration parameter did not have much influence on performance deviations. This is consistent with the conclusions of the two-source configuration and validates the use of a bufferless model that is oblivious to this parameter.

Another aspect that was investigated in the two-source case was the amount of additional bandwidth needed to ensure that the type 2 source saw the target loss. We carry out a similar investigation in the many-source case, and the results are reported in Figure 4. The figure shows that across all the scenarios of Figure 3, the maximum amount of additional bandwidth needed is only $8 \%$, as compared to levels in excess of $40 \%$ for some of the two-source scenarios. This further confirms our earlier intuition regarding the benefits of larger scale systems towards ensuring consistent performance across users. However, note that in absolute value, the additional bandwidth required to adequately accommodate a low utilization, high peak rate source remains high.

Finally, a last aspect that we investigated was the sensitivity of our findings to the fact that there was only a single type 2 source. In particular, as the proportion of type 2 sources increase, so will their impact on the overall loss probability, which should then improve their ability to attract sufficient additional resources to ensure that they experience the desired loss target. Clearly, no deviation would exist if all the background traffic were made of only type 2 sources. Our goal is, therefore, to determine how quickly this happens as we increase the proportion of type 2 sources. To test this, we select the parameters of type 2 sources to be $R_{2} / R_{1}=\rho_{1} / \rho_{2}=200$, progressively increase the traffic contributed by the type 2 sources to the background while keeping the total average background traffic fixed. 
The results of this experiment are displayed in Figure 5.
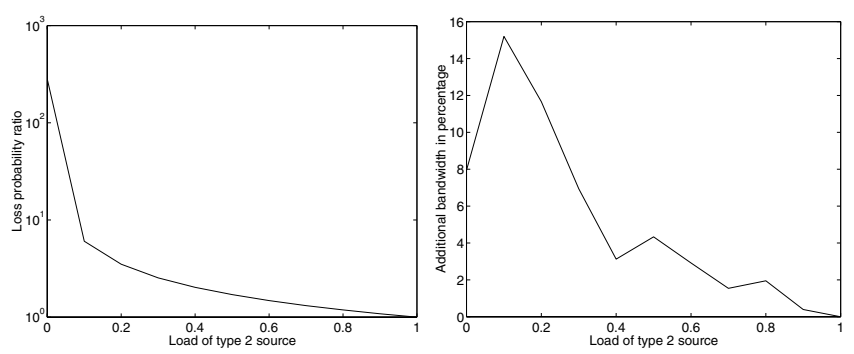

Fig. 5. Increasing the Background Load Contributed By Type 2 Sources.

The figure shows the behavior of the loss probability ratio as the proportion of type 2 sources in the background increases (lhs), as well as the amount of additional bandwidth needed to ensure that type 2 sources experience the desired loss target (rhs). From the figure, we see that as expected, increasing the number of type 2 sources helps decrease the magnitude of the performance deviation they experience. However, while there is a sharp initial decrease as the number of type 2 sources increases from one, the slope rapidly levels off so that even when the type 2 sources make up about $50 \%$ of the traffic they still experience a loss probability that is twice as large as that of type 1 sources. This behavior is further confirmed by the relatively slow convergence of the amount of additional bandwidth needed. This points to the fact that merging two service classes used to carry significantly different traffic in terms of peak rate and utilization, should probably be avoided.

To summarize, what we've seen is that in the many ON-OFF sources case, the main factor responsible for causing significant deviations in performance is a combination of high peak rate and low utilization. This is different from what is observed in the two-source case, where utilization alone can induce significant deviations. The reason for this is that in order for an individual source to experience losses that differ significantly from the overall losses, it needs to not only contribute to congestion, but do it in a way that does not trigger the allocation of sufficient additional resources. In the case of two or a small number of sources, because each source has an important impact on congestion, even when they have similar peak rates, decreasing the utilization of one of them decreases the overall frequency of congestion periods and, therefore, improves the overall performance but not its own. In contrast, when there are many sources, decreasing solely the utilization of an individual source has only minor impact on both the overall performance and its own. In order for performance deviations to occur and remain undetected at the aggregate level, the peak rate of the source needs to be increased while its utilization is decreased to ensure that the resulting additional losses do not substantially contribute to the overall losses.

In the next sub-section, we investigate whether the conclusions reached for many ON-OFF sources also apply to periodic sources. Intuitively, we expect that the "smoother" nature of periodic sources plus the large number of sources, should result in relatively small performance deviations across all scenarios.

\section{B. Many Periodic Sources}

The configuration used in this section consists of 1000 type 1 sources with period $D_{1}$ and a single type 2 source with period $D_{2}$. The ratio $D_{2} / D_{1}$ is then varied from 40 to 200 . The link load is initially fixed at 0.7 and the buffer size is set to ensure an overall loss probability of $10^{-4}$ for all configurations. The link load is then decreased until the type 2 source also achieve the $10^{-4}$ target loss probability.

\begin{tabular}{|l|c|c|c|c|c|}
\hline$D_{2} / D_{1}$ & 40 & 80 & 120 & 160 & 200 \\
\hline$P_{L}^{2} / P_{L}$ & 1.0235 & 1.0238 & 1.0239 & 1.0240 & 1.0240 \\
\hline$\Delta C / C \%$ & 0.0981 & 0.0997 & 0.1004 & 0.1004 & 0.1004 \\
\hline
\end{tabular}

TABLE I

IMPACT OF SOURCE PERIOD: THE MANY SOURCE CASE.

The results of those experiments are reported in Table I. They confirm our expectation that the large number of sources combined with the relatively smooth nature of periodic traffic results in only minor differences. Differences in loss probability are of the order of $2 \%$ and the amount of bandwidth required to bring the type 2 source on par with type 1 sources is at most $0.1 \%$ of the allocated bandwidth. Intuitively, this is because when the number of sources is large, packet arrival epochs are more likely to be evenly distributed, which makes for smaller queue length variations and, therefore, a lower sensitivity to the frequency at which the queue is sampled. Hence, the type 2 source will sample the queue in almost the same way as the type 1 sources. More formally, this can be deduced from the well-known PASTA result, as the many type 1 sources result in an overall arrival process that approaches Poisson, so that type 1 arrivals sample the queue at random times. Similarly, increasing the period of the type 2 source will also have it sample the queue at essentially a random time. Hence the two types of sources should see similar queue statistics and performance.

The main significance of this finding is that it lends some validity to the use of aggregate QoS solutions to support constant rate services. Clearly, there are aspects that the periodic model does not capture, e.g., how interactions between flows affect the periodic nature of the traffic as it traverses the network (see [2], [10], [14] for relevant investigations of this issue). However, it helps address an important issue, namely, when relying on such a service model, whether differences in rate affect individual performance. The answer, based on the models we have developed, is that this is not the case, at least not when many such sources are aggregated into a common service class. Note that the answer obtained in Section IV-B was different in that it pointed out the potential for significant differences in the two (few) source case, when one of the sources had a much longer period. The transition between these two behaviors is investigated in greater details in Section VI.

\section{INTERMEDIATE CONFIGURATIONS}

Because of the differences that were observed between the two-source configurations and the many-source ones, it is of interest to gain some understanding into how fast one transitions from one set of behaviors to the other. Note that the differences between the two configurations were not unexpected. 
As a matter of fact, the two-source and many-source cases were chosen because of both their analytical tractability and the fact that we expected them to provide results that would applicable to "small" and "large" systems respectively. What we wish to undertake in this last section, is to get a better understanding on what exactly does "small" and "large" mean.

The approach we take is to test by means of simulations a number of "intermediate" scenarios. The first scenario is for ON-OFF sources. In particular, the number of background (type 1) sources in the system is increased from 1 to 100. When there's only one type 1 source, its parameters are: $R_{1}=$ $10^{8} \mathrm{bits} / \mathrm{sec}, b_{1}=0.005 \mathrm{sec}$, and $\rho_{1}=0.5$. When the number of type 1 sources increases, $b_{1}$ and $\rho_{1}$ remain fixed but $R_{1}$ is decreased so that the total mean rate of the background is kept constant. As before, when considering differences in source traffic parameters, we choose a large ratio of 200 between the parameters of the two types of sources. Note that simulations were carried out for both bufferless and buffered systems (the buffer size was again 0.5 Mbits). Because results were essentially similar, we only report those of the buffered system simulation. In addition, for the sake of brevity, we focus on scenarios for which the behaviors of the two-source and many-source scenarios differ. In particular, we considers cases that involve differences in $\rho$ and in both $R$ and $\rho$.

\begin{tabular}{|c|c|c|c|c|c|c|}
\hline \multirow{3}{*}{$\frac{\rho_{1}}{\rho_{2}}=200$} & $N_{1}$ & 1 & 10 & 20 & 50 & 100 \\
\cline { 2 - 7 } & $P_{L}^{2} / P_{L}$ & 100 & 3.3 & 1.8 & 1.1 & 1.4 \\
\cline { 2 - 7 } & $\Delta C / C(\%)$ & 23.9 & 2.5 & 0.9 & 0.1 & 0.07 \\
\hline \multirow{3}{*}{$\frac{\rho_{1}}{\rho_{2}}=200$} & $N_{1}$ & 1 & 10 & 20 & 50 & 100 \\
\cline { 2 - 7 }$\frac{R_{2}}{R_{1}}=200$ & $P_{L}^{2} / P_{L}$ & 2 & 10.4 & 19.5 & 44 & 81 \\
\cline { 2 - 7 } & $\Delta C / C(\%)$ & 0.02 & 0.8 & 1.7 & 4.4 & 9.1 \\
\hline
\end{tabular}

TABLE II

INTERMEDIATE ON-OFF SCENARIOS.

The results are reported in Table II. Note that the 95\% confidence intervals for most simulations are still relatively large (of the order of $40 \%$ ), which explains some of the "irregularities" seen in the table. We draw a number of conclusions from the results in the table.

First, when the two types of sources differ only in their utilization, the convergence to the many-source results is rather rapid. For example, to rely on the many-source results, a number of only 50 background sources is sufficient and by allocating an additional $0.1 \%$ of bandwidth, both types of sources would experience the desired target loss probability. This means when sources differ only in their mean rate and not their peak rate, e.g., because they have the same access speed constraints, aggregating even a relatively small number of (ON$\mathrm{OFF}$ ) sources with different mean rates in the same service class is reasonable.

The situation is somewhat different when dealing with sources that differ in both their utilization and peak rate. In such case, the transition from the two-source behavior to the many-source one is much more progressive. More specifically, the behavior of the two-source case prevails for some time, e.g., until a background traffic of about 10 to 20 sources, and then
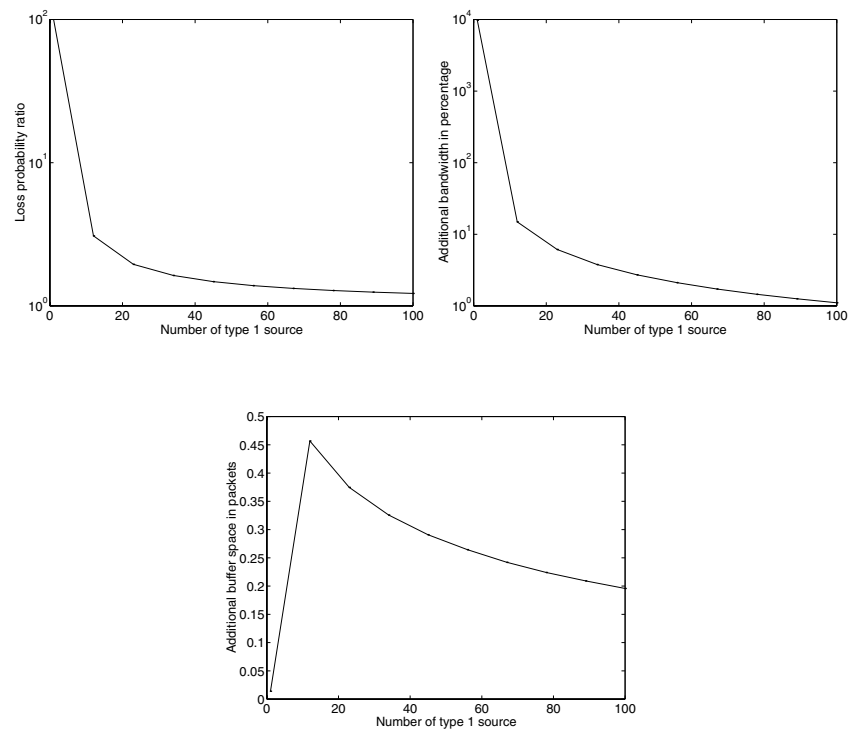

Fig. 6. Intermediate Scenarios for Periodic Sources.

slowly transitions to the many-source behavior. However, even if it takes a large number of sources before reaching the very large performance deviations that have been described in the many-source regime in Section V-A, significant differences in performance can already be observed even for a relatively small number of sources. Specifically, even with only 10 sources the loss probability ratio is already greater than 10 . This implies that aggregating even a small number of sources that differ in both their peak rate and utilization can be dangerous.

The second intermediate configuration that was tested corresponds to the $\sum_{j}\left(N_{j} \cdot D_{j}+D_{i}\right) / D / 1$ queue of Section V-B. In this experiment, we do not rely on simulations and still use the formula 8 and 9 in Section III-D. We vary the number of type 1 sources from 1 to 100 , while the ratio $D_{2} / D_{1}$ remains fixed at 200. The loss probability ratio and the additional bandwidth needed to satisfy the performance requirements of the type 2 source are given in Figures 6(a) and 6(b), respectively.

As can be seen from the figure, the behavior of the manysource scenario is reached rather rapidly, and a background traffic of 100 sources is more than sufficient for that purpose. Figures 6(c) also shows another interesting result, namely that across all configurations performance deviations can be readily handled through the addition of a single packet buffer. This highlights the sensitivity of deviation to buffer size in systems where congestion occurs at the packet level and further validates the ability to provide performance assurance using aggregation, when dealing with this kind of sources.

\section{CONCLUSION}

This paper is concerned with an environment where QoS is provided using coarse mechanisms such as service classes, which are used to aggregate many individual flows. In addition, we also assume that service guarantees are provided through provisioning and the monitoring of aggregate performance measures for each service class. In particular, our focus is on loss guarantees. In that context, our goal is to determine if 
and when aggregate performance measures are a good predictor of individual ones, and to identify scenarios where this may not hold. For those, we seek to determine the combinations of traffic parameters responsible for such deviations. We then use them to formulate guidelines on which types of traffic can be safely aggregated in the same service class.

Our approach is analytical in nature. We rely on two source models, Markov ON-OFF and periodic, that we feel provide an adequate coverage of the different possible environments. We also investigate two limiting configurations, a two-source model and a many-source model, that are expected to help us characterize the likely range of possible behaviors.

The contributions of this paper are two-fold. First, we derive a number of analytical models that allow the evaluation of individual loss probabilities in environments that only provide aggregate guarantees. Those models build on previous works but do represent new contributions. In particular, the results of Sections III-A and III-B provide some simple but yet useful insight into the impact of individual traffic parameters in the two-source case. Section III-D also provides new results.

Second, we identify a number of cases and traffic parameters that introduce significant deviations between individual and aggregate performance, and use those results to provide guidelines for avoiding such situations. In particular, we showed that when service classes are used to aggregate only a small number of users, the utilizations of individual users were a key factor and one should avoid multiplexing users with significantly different utilizations. This held for both the ON-OFF and the periodic models. In contrast, when the number of users aggregated is large, it is desirable to avoid multiplexing flows that differ greatly in both their peak rate and utilization, at least in environments where provisioning has some built-in assumption regarding multiplexing efficiency, i.e., for which the $\mathrm{ON}$ OFF model is relevant. If a more conservative, e.g., peak rate based, provisioning is used, then multiplexing flows with different traffic characteristics (periods) is reasonably safe, as long as the number of flows that are aggregated in the service class is sufficiently large.

At last, the differences that were identified between the provisioning environments corresponding to the ON-OFF and periodic models, also carry over in how fast the many-source model becomes applicable. In particular, in the periodic model the impact of rate differences disappears rapidly as the number of sources increase. However, the same does not hold for the ONOFF model, where a significantly larger number of sources is needed before the many-source results hold, at least when the sources differ in both their peak rate and utilization.

We believe that the results reported in this paper can provide useful data points and guidelines regarding the capabilities and limitations of aggregate QoS models. Obviously, the results of this paper represent only a starting point, and we are currently investigating a number of extensions. In particular, one aspect that is currently being investigated is the evaluation of actual loss performance deviation when aggregating more realistic traffic sources, e.g., voice sources and/or video sources and the effectiveness of using the models described in this paper, i.e, either ON-OFF sources or periodical sources, to predict the magnitude or at a minimum the presence of such deviations.

\section{REFERENCES}

[1] D. Anick, D. Mitra, and M. M. Sondhi. Stochastic theory of a datahandling system with multiple sources. Bell Sys. Tech. Journal (BSTJ), 61(8):1871-1894, October 1982

[2] J. C. R. Bennett, K. Benson, A. Charny, W. F. Courtney, and J.-Y. Le Boudec. Delay jitter bounds and packet scale rate guarantee for Expedited Forwarding. In Proceedings INFOCOM'2001, Anchorage, Alaska, April 2001.

[3] S. Blake, D. Black, M. Carlson, E. Davies, Z. Wang, and W. Weiss. An architecture for differentiated services. Request For Comments (Proposed Standard) RFC 2475, IETF, December 1998.

[4] B. Davie, (Ed.), A. Charny, F. Baker, J. Bennet, K. Benson, J.-Y. Le Boudec, A. Chiu, W. Courtney, S. Davari, V. Firoiu, C. Kalmanek, K.K. Ramakrishnam, and D. Stiliadis. An expedited forwarding PHB. Internet draft (work in progress), IETF, April 2001. (draft-ietf-diffservrfc2598bis-01.txt).

[5] R. J. Gibbens and P. J. Hunt. Effective bandwidths for the multi-type UAS channel. QUESTA, 9(1-2):17-27, October 1991.

[6] R. J. Gibbens, F. P. Kelly, and P. B. Key. A decision-theoretic approach to call admission control in ATM networks. IEEE J. Select. Areas Commun., SAC-13(6):1101-1113, 1995.

[7] M. Grossglauser and D. N. C. Tse. A framework for robust measurementbased admission control. In Proceedings SIGCOMM'97, Cannes, France, August 1997.

[8] R. Guérin, H. Ahmadi, and M. Naghshineh. Equivalent capacity and its application to bandwidth allocation in high-speed networks. IEEE J. Select. Areas Commun., SAC-9(7):968-981, September 1991.

[9] R. Guérin and V. Peris. Quality-of-service in packet networks: Basic mechanisms and directions. Computer Networks, 31(3):169-179, February 1999 .

[10] R. Guerin and V. Pla. Aggregation and conformance in Differentiated Service networks: A case study. ACM SIGCOMM Computer Communication Review (CCR), 31(1):21-32, January 2001.

[11] L. Gün and R. Guérin. Bandwidth management and congestion control framework of the broadband network architecture. Computer Networks and ISDN Systems, 26(1):61-78, September 1993.

[12] S. Jamin, P. Danzig, S. Shenker, and L. Zhang. A measurement- based admission control algorithm for integrated services packet networks. IEEE/ACM Trans. Networking, 5(1):56-70, February 1997.

[13] V. Kumar, T. V. Lakshman, and D. Stiliadis. Beyond best-effort: Router architectures for the differentiated services of tomorrow's internet. IEEE. Commun. Mag., 36(5):135-150, May 1998.

[14] L. Massoulié. Larges deviations orderings of point processes in some queueing networks. Queueing Systems, 28(4):317-335, 1998.

[15] D. Mitra. Stochastic theory of a fluid model of producers and consumers coupled by a buffer. Adv. Appl. Prob, 20:646-676, 1988.

[16] I. Norros and J. Virtamo. Who loses cells in the case of burst scale congestion. In Proceedings of the 13th Intl. Teletraffic Congress (ITC-13), pages 59-64, Copenhagen, Denmark, June 1991.

[17] J. W. Roberts, U. Mocci, and J. Virtamo, editors. Broadband Network teletraffic - Final Report of Action COST 242, volume 1155. SpringerVerlag, 1996

[18] J. W. Roberts and J. Virtamo. The superposition of periodic cell arrival streams in an ATM multiplexer. IEEE. Trans. Commun., 39(2):298-303, February 1991.

[19] T. E. Stern and A. I. Elwalid. Analysis of separable Markov-modulated rate models for information-handling systems. Adv. Appl. Prob., 23:105139, 1991.

[20] I. Stoica, S. Shenker, and H. Zhang. Core-stateless fair queueing: A scalable architecture to approximate fair bandwidth allocations in high-speed networks. In Proceedings of SIGCOMM'98, pages 118-130, Vancouver, B.C., Canada, September 1998.

[21] I. Stoica and H. Zhang. Providing guaranteed service without per flow management. In Proceedings of SIGCOMM'99, pages 81-94, Boston, MA, September 1999

[22] J.T. Virtamo and J. Roberts. Evaluating buffer requirements in an ATM multiplexer. In Proceedings of GLOBECOM'89, pages 1473-1477, Dallas, TX, 1989.

[23] Y. Xu and R. Guérin. Individual versus aggregate QoS: A loss performance study. Technical report, University of Pennsylvania, July 2001. (Available at http://seas.upenn.edu/ $/$ guerin/).

[24] Z.-L. Zhang, Z. Duan, L. Gao, and Y.T. Hou. Decoupling QoS control from core routers: A novel bandwidth broker architecture for scalable support of guaranteed services. In Proceedings of SIGCOMM'2000, pages 71-83, Stockholm, Sweden, August 2000. 